\title{
Commentary
}

\section{Cognitive neuroscience of ownership and agency is}

\author{
Lars Schwabe *, Olaf Blanke \\ Laboratory of Cognitive Neuroscience, Brain Mind Institute, Station 15, Ecole Polytechnique Fédérale de Lausanne (EPFL), \\ 1015 Lausanne, Switzerland \\ Department of Neurology, University Hospital, Geneva, Switzerland
}

\section{Introduction}

The paper by Tsakiris et al. reviews an important series of recent studies that have investigated body ownership and agency using techniques from experimental psychology and neuroimaging. The reviewed data highlight the importance of multisensory as well as sensori-motor signals for both phenomena and tackle the fascinating issue of how humans' everyday (mostly unconscious) bodily experiences of ownership and agency have been correlated with behavior and neural activation in controlled experiments. This is complemented by an introduction to important philosophical issues raised by the neuroscience of ownership and agency. The paper also reviews recent findings on the related topic of self-other discrimination. In what follows we have focussed on open questions for future research that seem important in order to build a cognitive science and cognitive neuroscience of bodily experience as well as bodily perception and cognition.

\section{Towards neurobiological definitions}

Tsakiris et al. review evidence that suggests that ownership and agency are pre-reflective experiences that are likely to be generated by low-level sensori-motor processes. But how exactly are ownership and agency produced by these processes? What are the underlying neural mechanisms and their dynamical and computational principles? What are the neurological conditions where these processes break down? We note that at present ownership and agency are far from being well-defined concepts. Despite the importance of recent efforts reviewed by Tsakiris et al. (2007), we believe phenomena both need to be further defined in behavioral and neurobiological terms, as in the case of visual perception. It seems well grounded that a combination of afferent sensory signals and efferent motor signals, as Tsakiris et al. suggest, are important sources for both phenomena, yet predicted and actual sensory feedback, as well as prediction error signals will likely have to be included as well. The lack of detailed computational models of ownership and agency formulated in terms of afferent sensory and efferent motor signals further complicates their anchoring to neuronal activations as measured in neuroimaging studies, although the review has indicated several interesting directions.

\footnotetext{
Commentary on Tsakiris, M., Schütz-Bosbach, S., \& Gallagher, S. (2007). On agency and body-ownership: Phenomenological and neurocognitive reflections. Consciousness and Cognition, 16, 645-660.

* Corresponding author. Address: Laboratory of Cognitive Neuroscience, Brain Mind Institute, Station 15, Ecole Polytechnique Fédérale de Lausanne (EPFL), 1015 Lausanne, Switzerland. Fax: +41 216939625.

E-mail address: lars.schwabe@epfl.ch (L. Schwabe).
} 
For example, the authors review a study by Farrer et al. (2003), where the visual feedback for self-generated movements was varied systematically. When the visual feedback was incongruent, the authors noted that the brain has to deal with two types of errors, i.e., a discrepancy between afferent visual signals and afferent proprioceptive signals or between afferent visual signals and efferent motor signals. Additional central errors may arise and have been argued to be of key importance in agency. Afferent visual signals, afferent proprioceptive signals, and efferent motor signals, are also compared with predicted sensory consequences using the efferent motor signals (Chapman, Bushnell, Miron, Duncan, \& Lund, 1987; Voss, Ingram, Haggard, \& Wolpert, 2006). Until latter errors as well as intersensory and sensori-motor errors are distinguished in greater detail, functional interpretations of behavioral and neuroimaging studies as well as implications for philosophy will remain ambiguous.

\section{Neural substrates}

Another point for future research on ownership and agency relates to providing causal evidence for the reviewed distinct contributions of afferent and efferent signals. Such evidence could be searched for in clinical conditions characterized by abnormal sensori-motor and/or multisensory processing. The authors review recent work in neurological patients with damage to large myelinated somatosensory fibers in the peripheral nervous system leading to a selective deficit in signaling of somatosensory information (Fourneret, Paillard, Lamarre, Cole, \& Jeannerod, 2002; Gallagher \& Cole, 1995). Despite the importance of these patient studies, it will be useful to carry out comparative analyses in other neurological patients suffering, for example, from damage to peripheral motor fibers. Other research on agency has focussed on psychiatric patients with schizophrenia suffering from so-called delusions of agency (Daprati et al., 1997; Farrer et al., 2004; Spence et al., 1997). The research on agency therefore investigated contributions of the peripheral somatosensory nervous system or of high-level cortical motor systems. Surprisingly, the many intermediate stages of processing are almost unexplored in neurological patients. This is unfortunate because the neuroimaging data reviewed by Tsakiris et al. suggest the importance of several areas such as temporo-parietal cortex, premotor cortex, insula, and primary somatosensory cortex for agency and ownership (Ehrsson, Spence, \& Passingham, 2004; Farrer et al., 2003, 2004; Spence et al., 1997; Tsakiris, Hesse, Boy, Haggard, \& Fink, 2006). Performance and experience with respect to agency and ownership of neurological patients with selective damage to any of these structures might reveal distinct contributions within the many layers of ownership and agency.

\section{Neuropsychology of ownership and agency}

Even more importantly, several neurological conditions have been described over the last 60 years with profound deficits in ownership. These conditions include somatoparaphrenia (e.g., Gerstmann, 1942; Moro, Zampini, \& Aglioti, 2004), asomatognosia (e.g., Arzy, Petit, Landis, \& Blanke, 2006; Critchley, 1953), and the alien hand syndrome (e.g., Brion \& Jedynak, 1972). Patients with somatoparaphrenia claim either that their contralesional arm belongs to another person or that another person's arm belongs to them (Gerstmann, 1942). Somatoparaphrenic ownership is thus characterized by the misattribution of another person's upper extremity (as in the RHI) or the misattribution of one's own upper extremity. What is the performance of such patients in ownership- and agency-related tasks that Tsakiris et al. have reviewed (if their motor weakness allows testing)? Bodily experience and behavior in such patients will probably differ from healthy subjects and performance is likely to differ for contra- and ipsilesional hands. Asomatognosia is also characterized by a profound disturbance of bodily experience and such patients report not feeling or not seeing contralesional body parts such as an arm or hand (Arzy et al., 2006) suggesting the possibility of preserved arm ownership without feeling or seeing one's arm. While brain damage in patients with somatoparaphrenia or asomatognosia often includes temporo-parietal cortex, insula, and/or premotor cortex different brain structures including medial parietal cortex and the corpus callosum are affected in patients with the alien hand syndrome (Brion \& Jedynak, 1972). These patients suffer from abnormal ownership (under somatosensory conditions) and do not recognize their own left hand when holding it in their right hand, but do so when seeing it. They have normal visual, tactile, proprioceptive, and motor functions. To summarize, investigations of abnormal ownership and 
(probably agency) in patients with these three conditions as well as patients with damage to some of the areas suggested to be involved in ownership and agency should apply some of the experimental paradigms reviewed by Tsakiris et al. This will allow to investigate hemispheric specialization, body-part specificity, and top-down effects of ownership and agency (Bottini, Bisiach, Sterzi, \& Vallar, 2002), as well as other factors that are not easily investigated in healthy subjects.

\section{From anarchic hand to bimanual agency and intention}

Anarchic hand (Feinberg, Schindler, Flanagan, \& Haber, 1992; Goldberg, Mayer, \& Toglia, 1982 ${ }^{1}$ ) and diagonistic dyspraxia (Akelaitis, 1945; Tanaka, Yoshida, Kawahata, Hashimoto, \& Obayashi, 1996) are two other fascinating and relevant neurological conditions for the study of ownership and agency. They are due to frontal and/or callosal damage and are characterized by unimanual or bimanual motor deficits and spontaneous non-intentional movements and are classically described as deficits of volition and intention. One could expect ownership and agency to be normal in such patients. However, experimental work in these conditions is needed to help disentangling intention from agency and ownership (Jeannerod, 2006). Goldberg et al. (1982) notes about a 63-year-old right-handed female patient with anarchic hand syndrome (due to left medial frontal infarction) that "the patient had picked up a pencil and had begun scribbling with the [affected] right hand. When her attention was directed to this activity, she reacted with dismay, immediately withdrew the pencil, and pulled the right hand to her side using the [normal] left hand. She then indicated that she had not herself initiated the original action of the right arm. She often reacted with dismay and frustration at her inability to prevent these unintended movements of the right arm. She experienced a feeling of dissociation from the actions of the right arm, stating on several occasions that "it will not do what I want it to do". Thus, one could speculate that the experience of being the author of her right dominant hand movements is disturbed in this patient, while agency for left hand movements seems normal. This suggests distinct and lateralized mechanisms of agency. Anarchic agency (and ownership) will probably differ for both hands. Moreover, the causing and the intending of an action both seem to be impaired in patients with anarchic hand syndrome.

Other lesions in this area may lead to diagonistic apraxia, a disorder of bimanual action that is defined as abnormal motor behavior of the left hand in conditions of voluntary movements of the right hand. Tanaka et al. (1996) describe a patient who "when his right hand was trying to grasp a target [...], his left hand would thrust itself into the field and grasp the target first, against his will. Or while he was trying to remove his underpants with his right hand, his left hand would suddenly reach over and raise the underpants. When he was picking up his trousers from the floor with the right hand, the left hand would simultaneously begin to unbutton his shirt." Despite the recent advances in the cognitive neuroscience of ownership and agency both latter neurological conditions leave the experimenter startled suggesting lateralized mechanisms as well as bimanual mechanisms of ownership, and agency, and calling for more complex models of ownership and agency. We further suggest that abnormal mechanisms of agency need to be distinguished experimentally from those of intention in these conditions.

\section{Computational neuroscience}

In order to model the interactions between ownership and agency (and intention), a first step will be their anchoring to sensori-motor processes as suggested by Tsakiris et al. (2007), as computational models of sensori-motor processes have already been developed and tested (Wolpert, Ghahramani, \& Jordan, 1995). An essential ingredient of these models is the use of so-called "forward models", which the brain may use to estimate the current configuration of the limbs based on efferent copies of motor commands, previous estimates, and afferent sensory signals. The figure shows a sketch of such a model. Here, a motor forward model utilizes an efferent copy of a motor command in order to predict the state of limbs before the motor command has been executed and sensory feedback received. Sensory forward models, however, may use the very same signal

\footnotetext{
${ }^{1}$ Note that there are inconsistencies with respect to the terms anarchic hand and alien hand. Here, we follow the initial French terminology introduced by Brion and Jedynak (1972) for alien hand ("la main etrangère") without non-intentional motor behavior, as opposed to anarchic hand ("la main capricieuse") that is always associated with non-intentional motor behavior; see text).
} 
in order to predict the sensory consequences of the motor command, which can then be compared to actual sensory inputs. Suppression of sensory inputs during active movement (Chapman et al., 1987; Voss et al., 2006) could be explained in this way. Moreover, one could also postulate that agency might rather be related to the proper matching of the predictions of sensory forward models and the actual sensory inputs as reflected by a vanishing error signal. Conversely, the loss of agency might be identified with an error signal triggered, for example, during incongruent visual feedback. In the same way, inter-sensory matches and mismatches within the sensory systems (Fig. 1) might be identified with ownership and its loss when the discrepancy between the afferent visual and proprioceptive signals exceeds a certain size.

Does this application of computational models of sensori-motor processes to ownership and agency fully capture both phenomena? Tsakiris et al. make two important points. First, they emphasize that ownership might be more than mere multisensory stimulation. For example, during the RHI (Botvinick \& Cohen, 1998), where the unseen real hand and the seen rubber hand are touched synchronously, subjects experience that the felt touch is caused by the seen touch, and that this correlation may then create the feeling "as if the rubber hand is my hand". This distinction between the multisensory stimulation (the causes) and the resulting self-attribution of the rubber hand (the effect) is important. But one could also conclude that multisensory representations of the afferent inputs could account for the perceptual experience of the seen touch causing the felt touch, but not for the feeling of ownership of the fake hand. We like to point out that already the computation of unisensory representations is a complex dynamic process involving the interaction of afferent feedforward signals and top-down predictions (for an example of the visual system: Rao \& Ballard, 1999). The computation of multisensory representations certainly involves those and even more complex interactions and crosstalk between modalities and levels of processing. Currently, we do not know enough about these processes or multisensory body representations. Studies modeling the computation of these representations will be an important step towards developing quantitative notions of ownership and agency.

Second, Tsakiris et al. discuss the interesting finding that active movements might modulate body ownership. Tsakiris, Prabhu, and Haggard (2006) have shown that the proprioceptive drift during the RHI is specific

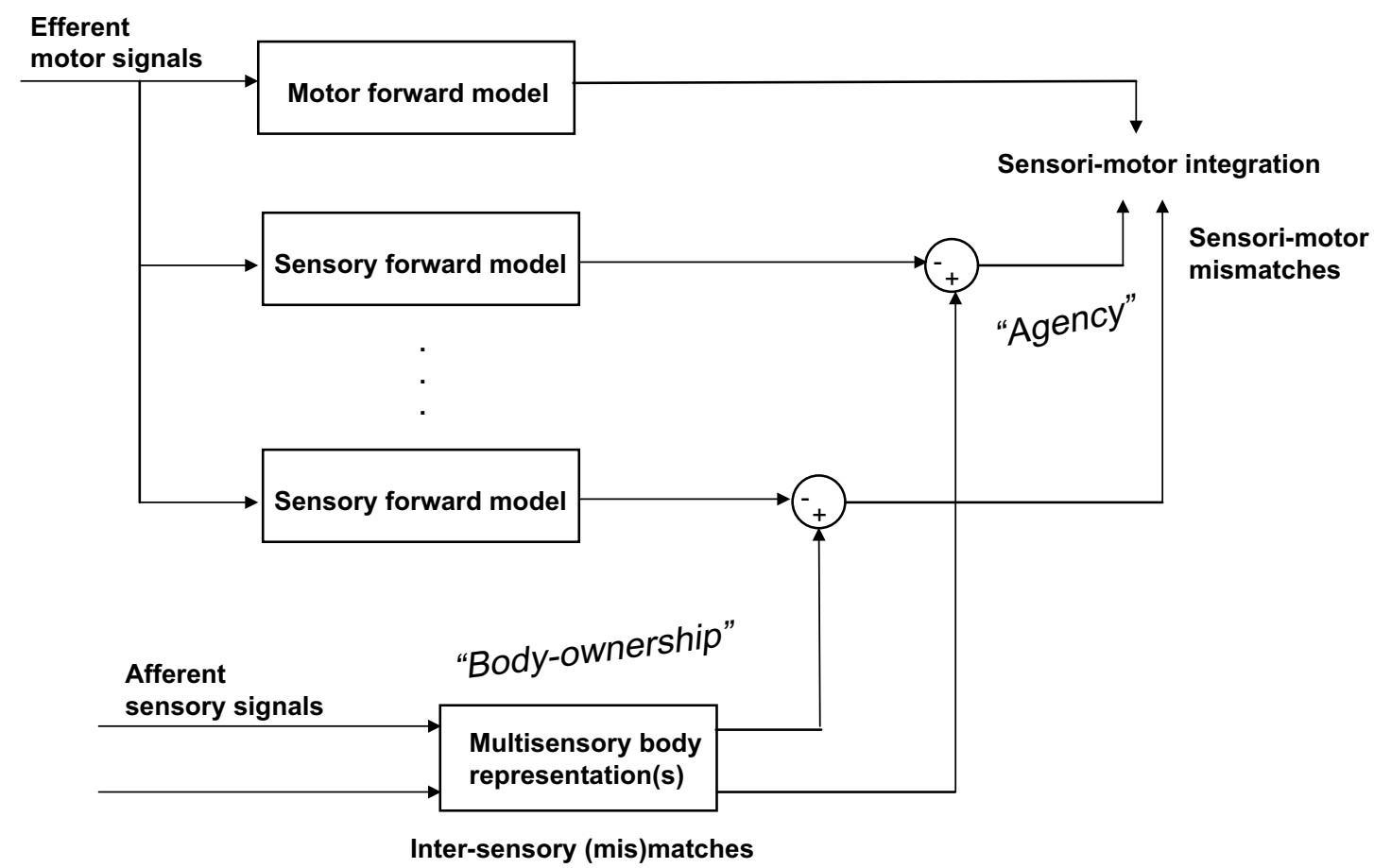

Fig. 1. Simplified sketch of a model for sensori-motor and multisensory processes and their role in ownership and agency. Ownership is linked to inter-sensory matches of afferent signals. Agency is identified with a vanishing error in predicting the sensory consequences of intentional motor actions (for further detail see text). 
to single digits when no voluntary movements are involved. More importantly, when the digit is actively moved during stroking, the proprioceptive drift extends to the entire hand. In other words, active movements seem to modulate the illusory localization of body parts beyond the stimulated body parts. Within the sketched model (Fig. 1), this would mean that the efferent copy of the motor command or the error signal itself affects multisensory body representations in more complex ways than just suppressing the sensory representations of the moving body part. Tsakiris et al. suggest that this interaction of signals that reflects active movements and multisensory body representations could serve to integrate the sensory representations of distinct body parts into a coherent representation of the hand.

\section{Conclusions}

It is an interesting idea that the brain may use signals corresponding to active movements in order to link the representations of fingers to each other and to build a representation of the entire hand. The study of more global own body representations (as opposed to single fingers) is relevant for the neuroscience of the bodily self as the bodily self generally refers to the subject of experience. The presence of such global own body representations is suggested by illusory own body perceptions called autoscopic phenomena due to damage to temporo-parietal or occipito-temporal cortex (Blanke, Landis, Spinelli, \& Seeck, 2004; Blanke \& Mohr, 2005; Brugger, Regard, \& Landis, 1997; Devinsky, Feldmann, Burrowes, \& Bromfield, 1989). They also suggest that comparable, but distinct, mechanisms of multisensory and sensori-motor integration exist for the global representation of the entire body as opposed to the representations for certain body parts. Multisensory mechanisms for such global ownership have recently been tested experimentally using virtual reality in healthy subjects. Lenggenhager, Tadi, Metzinger, and Blanke (2007) showed that illusory self-attribution and self-location can be induced for the entire body: During multisensory conflict participants considered a virtual body seen in front of them to be their own body and mislocalized themselves towards the virtual body, to a position outside their bodily borders. This finding extends previous data on ownership that have investigated body part ownership or the attribution and localization of a body part with respect to the global self, i.e., a part-to-whole relationship. Importantly, illusory global self-attribution and self-location to a position outside one's body shows that global ownership (or selfhood) can be dissociated from one's physical body position concordant with altered selfhood in neurological patients with autoscopic phenomena (Blanke et al., 2004). This differs from the RHI where this aspect of selfhood remained constant and only the attribution and localization of a stimulated finger or hand was manipulated (see also Ehrsson, 2007).

We conclude that the experimental behavioral paradigms and data reviewed by Tsakiris et al. make it now possible to study selective aspects of ownership and agency of fingers, arms, and bodies. These experimental findings should be tested and extended (1) to neurological patients with abnormal ownership and agency, (2) in neuroimaging studies, and (3) in modeling studies applying principles from computational neuroscience in order to build up the field of cognitive neuroscience of bodily experience, bodily perception and cognition as is already the case in vision research.

\section{References}

Akelaitis, A. J. (1945). Studies on the corpus callosum, IV. Diagonistic dyspraxia in epileptics following partial and complete section of the corpus callosum. The American Journal of Psychiatry, 101, 594-599.

Arzy, S., Petit, L., Landis, T., \& Blanke, O. (2006). Neural mechanisms of embodiment. Asomatognosia due to premotor cortex damage. Archives of Neurology, 63, 1022-1025.

Blanke, O., Landis, T., Spinelli, L., \& Seeck, M. (2004). Out-of-body experience and autoscopy of neurological origin. Brain, 127, $243-258$.

Blanke, O., \& Mohr, C. (2005). Out-of-body experience, heautoscopy, and autoscopic hallucination of neurological origin implications for neurocognitive mechanisms of corporeal awareness and self-consciousness. Brain Research. Brain Research Reviews, 50, $184-199$.

Bottini, G., Bisiach, E., Sterzi, R., \& Vallar, G. (2002). Feeling touches in someone else's hand. Neuroreport, 13, $249-252$.

Botvinick, M., \& Cohen, J. D. (1998). Rubber hands 'feel' touch that eyes see. Nature, 391, 756.

Brion, S., \& Jedynak, C. P. (1972). Troubles du transfert interhemispherique (callosal disconnection). A propos de trois observations de tumeurs du corps calleux: le signe de la main etrangere. Revue Neurologique (Paris), 126, 257-266.

Brugger, P., Regard, M., \& Landis, T. (1997). Illusory reduplication of one's own body: Phenomenology and classification of autoscopic phenomena. Cognitive Neuropsychiatry, 2, 19-38. 
Chapman, C. E., Bushnell, M. C., Miron, D., Duncan, G. H., \& Lund, J. P. (1987). Sensory perception during movement in man Experimental Brain Research, 68, 516-524.

Critchley, M. (1953). The parietal lobes. London: Edward Arnold.

Daprati, E., Franck, N., Georgieff, N., Proust, J., Pacherie, E., Dalery, J., et al. (1997). Looking for the agent: An investigation into consciousness of action and self-consciousness in schizophrenic patients. Cognition, 65, 71-86.

Devinsky, O., Feldmann, E., Burrowes, K., \& Bromfield, E. (1989). Autoscopic phenomena with seizures. Archives of Neurology, 46, 1080-1088.

Ehrsson, H. H., Spence, C., \& Passingham, R. E. (2004). That's my hand! Activity in premotor cortex reflects feeling of ownership of a limb. Science, 305, 875-877.

Ehrsson, H. H. (2007). The experimental induction of out-of-body experiences. Science, 317, 1048.

Farrer, C., Franck, N., Georgieff, N., Frith, C. D., Decety, J., \& Jeannerod, M. (2003). Modulating the experience of agency: A positron emission tomography study. NeuroImage, 18, 324-333.

Farrer, C., Franck, N., Frith, C. D., Decety, J., Georgieff, N., d'Amato, T., et al. (2004). Neural correlates of action attribution in schizophrenia. Psychiatry Reserach, 131, 31-44.

Feinberg, T. E., Schindler, R. J., Flanagan, N. G., \& Haber, L. D. (1992). Two alien hand syndromes. Neurology, 42, 19-24.

Fourneret, P., Paillard, J., Lamarre, Y., Cole, J., \& Jeannerod, M. (2002). Lack of conscious recognition of one's own actions in a haptically deafferented patient. Neuroreport, 13, 541-547.

Gallagher, S., \& Cole, J. (1995). Body schema and body image in a deafferented subject. Journal of Mind and Behavior, 16, 369-390.

Gerstmann, J. (1942). Problem of imperception of disease and of impaired body territories with organic lesions. Relation to body scheme and its disorders. AMA Archives of Neurology and Psychiatry, 48, 890-913.

Goldberg, G., Mayer, N. H., \& Toglia, J. U. (1982). Medial frontal cortex infarction and the alien hand sign. Archives of Neurology, 38, 683-686.

Jeannerod, M. (2006). Motor cognition. Oxford University Press

Lenggenhager, B., Tadi, T., Metzinger, T., \& Blanke, O. (2007). Video ergo sum: Manipulating bodily self-consciousness. Science, 317, 1096-1099.

Moro, V., Zampini, M., \& Aglioti, S. M. (2004). Changes in spatial position of hands modify tactile extinction but not disownership of contralesional hand in two right brain-damaged patients. Neurocase, 10, 437-443.

Rao, R. P., \& Ballard, D. H. (1999). Predictive coding in the visual cortex: A functional interpretation of some extra-classical receptivefield effects. Nature Neuroscience, 2, 79-87.

Spence, S. A., Brooks, D. J., Hirsch, S. R., Liddle, P. F., Meehan, J., \& Grasby, P. M. (1997). A PET study of voluntary movement in schizophrenic patients experiencing passivity phenomena: Delusions of alien control. Brain, 120, 1997-2011.

Tanaka, Y., Yoshida, A., Kawahata, N., Hashimoto, R., \& Obayashi, T. (1996). Diagonistic dyspraxia. Clinical characteristics, responsible lesion and possible underlying mechanism. Brain, 119, 859-873.

Tsakiris, M., Hesse, M. D., Boy, C., Haggard, P., \& Fink, G. R. (2006). Neural signatures of body ownership: A sensory network for bodily self-consciousness. Cerebral Cortex, in press, doi:10.1093/cercor/bhl131.

Tsakiris, M., Prabhu, G., \& Haggard, P. (2006). Having a body versus moving your body: How agency structures body-ownership Consciousness and Cognition, 15, 423-432.

Tsakiris, M., Schütz-Bosbach, S., \& Gallagher, S. (2007). On agency and body-ownership: Phenomenological and neurocognitive reflections. Consciousness and Cognition, 16, 645-660.

Voss, M., Ingram, J. N., Haggard, P., \& Wolpert, D. M. (2006). Sensorimotor attenuation by central motor command signals in the absence of movement. Nature Neuroscience, 9, 26-27.

Wolpert, D. M., Ghahramani, Z., \& Jordan, M. I. (1995). An internal model for sensorimotor integration. Science, $269,1880-1882$. 